\title{
Neural monitoring in endocrine neck surgery
}

\author{
Mustapha El Lakis ${ }^{1}$, Electron Kebebew ${ }^{2}$ \\ ${ }^{1}$ Endocrine Oncology Branch, National Cancer Institute, Bethesda, Maryland, USA; ${ }^{2}$ Department of Surgery, Stanford University, Stanford, \\ California, USA \\ Correspondence to: Mustapha El Lakis, MD. Endocrine Oncology Branch, National Cancer Institute, 10 Center Drive, Bethesda, MD 20892, USA. \\ Email: moustapha_lakis@hotmail.com. \\ Provenance: This is a Guest Editorial commissioned by Section Editor Xiao-li Liu (Deputy Director of Science and Education Department, \\ Department of Thyroid Surgery, China-Japan Union Hospital of Jilin University, Changchun, China). \\ Comment on: Kim HY, Liu X, Wu CE, et al. Future Directions of Neural Monitoring in Thyroid Surgery. J Endocr Surg 2017;17:93-103.
}

Submitted Mar 08, 2018. Accepted for publication Mar 14, 2018.

doi: $10.21037 /$ gs.2018.03.08

View this article at: http://dx.doi.org/10.21037/gs.2018.03.08

Recurrent laryngeal nerve (RLN) injury is the most dreaded complication in endocrine neck surgery. It can manifest as dysphonia, dysphagia, paroxysmal coughing, aspiration, or acute airway obstruction. A substantial proportion of patients $(80 \%)$ undergoing thyroid surgery may complain of voice changes (1). RLN injury has been ranked among the leading reasons for malpractice lawsuits. The true injury rate is underreported, and it is proportional to the rate of post-thyroidectomy laryngeal examinations. Surgery for thyroid cancer, especially reoperative and when it involves nodal dissection, has been identified as a significant risk factor for RLN palsy. In some reports, up to $25 \%$ of patients may sustain a transient laryngeal nerve injury following thyroid surgery, with persistent hoarseness occurring in $4 \%(2,3)$.

In 1938, Lahey and Hoover introduced the principle of routinely identifying the RLN during thyroid surgery, which eventually became the standard of care, resulting in a reduction in the rate of nerve palsy $(4,5)$. However, several studies indicated that visual inspection has a poor sensitivity in detecting nerve injury at the time of thyroid surgery, especially when RLN integrity was visually assured. In the 1970s, neuromonitoring was introduced with the premise of facilitating nerve identification (6) and, consequently, decreasing the rates of injury to the RLN and the external branch of the superior laryngeal nerve. Intraoperative nerve monitoring (IONM) offers a functional dynamic of evoked electromyography in response to nerve stimulation. Many techniques haven been described for conventional IONM. Intermittent IONM can be considered a major drawback of conventional IONM, as it helps identify the injury after its occurrence. Another IONM technique is continuous IONM (cIONM), which gives the surgeon continuous verification of electromyography responses-including amplitude and latency measures-through the operation. cIONM has been shown to be feasible, reliable, and safe for monitoring RLN status during thyroid surgery (7). Kim et al. have discussed in depth the two different techniques, along with their advantages and limitations, and recommended future directions (8).

IONM can provide information than sight alone during thyroid surgery. It helps (I) in neural mapping before visual nerve identification, (II) in prognostic information about the nerve function, (III) in localizing the injured nerve segment, and finally (IV) in decision making such as staged surgery if nerve injury is suspected.

The question is whether there is enough evidence in the literature to support routine IONM. A meta-analysis of six randomized controlled trials assessing the use of IONM in comparison to visual identification of the nerve, including 1,602 patients and 3,064 nerves at risk, showed no statistically significant decrease in the risk of temporary or permanent RLN injury (9). Similarly, a large meta-analysis, evaluating 64,699 nerves at risk, showed no superiority of IONM in comparison to visual nerve identification alone in terms of temporary or permanent RLN injury during thyroidectomy (10). On the other hand, a meta-analysis evaluating 36,487 at risk-nerves demonstrated a reduction in the incidence of transient RLN injury in patients undergoing IONM versus visual identification only; 
However, no difference in the incidence of permanent RLN injury was identified (11).

Despite these findings, interest in IONM has grown over the years. A survey of recently trained endocrine and head and neck surgeons revealed that the majority (95\%) routinely use IONM during thyroid surgery (12). However, it is not yet widely used. For instance, IONM was used in only $8.3 \%$ of patients who underwent thyroid surgery between 2010 and 2011 in Florida, New York, and Washington (13). That notwithstanding, IONM has been advised by multiple organizations, including the American Thyroid Association, the International Neural Monitoring Study Group, the American Academy of Otolaryngology and Head and Neck Surgery, and the American Head and Neck Society, to facilitate nerve identification and confirm nerve function $(4,14-16)$. They suggest it be used mainly in bilateral thyroid surgery, reoperative thyroid surgery, and surgery in the setting of existing RLN paralysis. They also note that neural monitoring is helpful in confirming intact neural function at the end of the surgery, which may impact the timing of the contralateral surgery, the need for tracheostomy, and the timing of discharge.

Surgeon's experience, proper training, and high-volume caseload continue to be the predictors of RLN injury in endocrine neck surgeries. IONM is evolving and is helpful in identifying nerve palsy and, hence, in staging endocrine neck surgeries. The widespread routine use of nerve monitoring among new generations of surgeons may provide further data to analyze the usefulness of the cIONM.

\section{Acknowledgements}

None.

\section{Footnote}

Conflicts of Interest: The authors have no conflicts of interest to declare.

\section{References}

1. Stojadinovic A, Shaha AR, Orlikoff RF, et al. Prospective functional voice assessment in patients undergoing thyroid surgery. Ann Surg 2002;236:823-32.

2. Lo CY, Kwok KF, Yuen PW. A prospective evaluation of recurrent laryngeal nerve paralysis during thyroidectomy. Arch Surg 2000;135:204-7.
3. Jeannon JP, Orabi AA, Bruch GA, et al. Diagnosis of recurrent laryngeal nerve palsy after thyroidectomy: a systematic review. Int J Clin Pract 2009;63:624-9.

4. Randolph GW, Dralle H, Abdullah H, et al. Electrophysiologic Recurrent Laryngeal Nerve Monitoring During Thyroid and Parathyroid Surgery: International Standards Guideline Statement. Laryngoscope 2011;121:S1-16.

5. Lahey FH, Hoover WB. Injuries to the recurrent laryngeal nerve in thyroid operations. Ann Surg 1938;108:545-62.

6. Flisberg K, Lindholm T. Electrical stimulation of the human recurrent laryngeal nerve during thyroid operation. Acta Otolaryngol Suppl 1969;263:63-7.

7. Phelan E, Schneider R, Lorenz K, et al. Continuous vagal IONM prevents recurrent laryngeal nerve paralysis by revealing initial EMG changes of impending neuropraxic injury: a prospective, multicenter study. Laryngoscope 2014;124:1498-505.

8. Kim HY, Liu X, Wu CE, et al. Future Directions of Neural Monitoring in Thyroid Surgery. J Endocr Surg 2017;17:93-103.

9. Sanabria A, Ramirez A, Kowalski LP, et al. Neuromonitoring in thyroidectomy: a meta-analysis of effectiveness from randomized controlled trials. Eur Arch Otorhinolaryngol 2013;270:2175-89.

10. Higgins TS, Gupta R, Ketcham AS, et al. Recurrent laryngeal nerve monitoring versus identification alone on post-thyroidectomy true vocal fold palsy: a meta-analysis. Laryngoscope 2011;121:1009-17.

11. Zheng S, Xu Z, Wei Y, et al. Effect of intraoperative neuromonitoring on recurrent laryngeal nerve palsy rates after thyroid surgery--a meta-analysis. J Formos Med Assoc 2013;112:463-72.

12. Marti JL, Holm T, Randolph G. Universal Use of Intraoperative Nerve Monitoring by Recently FellowshipTrained Thyroid Surgeons is Common, Associated with Higher Surgical Volume, and Impacts Intraoperative Decision-Making. World J Surg 2016;40:337-43.

13. Al-Qurayshi Z, Randolph GW, Alshehri M, et al. Analysis of Variations in the Use of Intraoperative Nerve Monitoring in Thyroid Surgery. JAMA Otolaryngol Head Neck Surg 2016;142:584-9.

14. Chandrasekhar SS, Randolph GW, Seidman MD, et al. Clinical practice guideline: improving voice outcomes after thyroid surgery. Otolaryngol Head Neck Surg 2013;148:S1-37.

15. Shindo ML, Caruana SM, Kandil E, et al. Management of invasive well-differentiated thyroid cancer: an American 
Head and Neck Society consensus statement. AHNS consensus statement. Head Neck 2014;36:1379-90.

16. Haugen BR, Alexander EK, Bible KC, et al. 2015 American Thyroid Association Management Guidelines for Adult Patients with Thyroid Nodules

Cite this article as: El Lakis M, Kebebew E. Neural monitoring in endocrine neck surgery. Gland Surg 2018;7(Suppl 1):S86-S88. doi: 10.21037/gs.2018.03.08 and Differentiated Thyroid Cancer: The American Thyroid Association Guidelines Task Force on Thyroid Nodules and Differentiated Thyroid Cancer. Thyroid 2016;26:1-133. 